\title{
Erratum: Role of Interventional Radiologist in the Management of Acute Pulmonary Embolism
}

\author{
William Bremer, MD ${ }^{1}$ Charles E. Ray Jr., MD, PhD, FSIR ${ }^{2}$ Ketan Y. Shah, MD² \\ 1 University of Illinois Hospital and Health Sciences System, \\ Address for correspondence William Bremer, MD, University of \\ Chicago, Illinois \\ 2Division of Interventional Radiology, Department of Radiology, \\ University of Illinois Hospital and Health Sciences System, \\ Illinois Hospital and Health Sciences System, Chicago, Illinois \\ (e-mail: wbremer2@uic.edu).
}

Chicago, Illinois

Semin Intervent Radiol 2020;37:62

ERRATUM

It has been brought to our attention that the FlowTriever device mentioned on pg. 68 is FDA approved in the above article in Seminars in Interventional Radiology, Volume 37, Number 1, 2020 (DOI: https://doi.org/10.1055/s-0039-3401841). Please see the following page for the correction. 
institution but are typically around 0.5 to $1 \mathrm{mg} /$ hour tPA per catheter if two catheters are used or 1 to $2 \mathrm{mg} /$ hour tPA if one catheter is deployed. A subtherapeutic heparin drip is infused during thrombolysis, with a partial thromboplastin time goal of approximately 40 to 60 seconds. ${ }^{18}$ Fibrinogen levels should be checked every 4 to 6 hours and if the level drops to less than 30 to $40 \%$ of the initial measurement, the rate of infusion may need to be reduced or stopped altogether. ${ }^{39}$ During thrombolysis, hemoglobin is trended serially and neurologic exams are performed frequently to assess for the development of hemorrhage. ${ }^{23}$ Endpoints for thrombolysis include improvement in respiratory status, RV strain evidenced by imaging or cardiac biomarkers, PA pressures, or pulmonary angiography.

\section{Device Options for Catheter-Directed Therapy}

A common technique for catheter-directed thrombolysis involves insertion of a multi-sidehole infusion catheter across the clot to maximize the surface area exposed to thrombolytic. ${ }^{23}$ In the setting of bilateral PE, two catheters are typically placed. Ultrasound-accelerated thrombolysis (USAT) is a variation of standard CDT which uses microsonic energy to assist with clot breakdown. Initially approved in 2004 by the FDA for use in the peripheral vasculature, the EKOS EndoWave Infusion Catheter System (EKOS Corporation, Bothell, WA) is a USAT system that was subsequently approved for use in the pulmonary circulation in $2014 .^{40}$ The catheter combines a multi-sidehole infusion catheter with a multi-element ultrasound core to deliver thrombolytic while the ultrasound core delivers high-frequency sound waves (-Fig.4). The sound waves cause disaggregation of fibrin and increase penetration of thrombolytic drug in in vitro studies. $^{41,42}$ Though many trials discussed below include use of the EKOS system, it remains unclear whether USAT is superior to standard CDT. ${ }^{43-45}$

One of the most basic techniques of mechanical thrombectomy is the rotating pigtail technique, utilized in a majority of patients worldwide undergoing CDT due to its simplicity and low cost. ${ }^{31}$ This technique involves rotation of a pigtail catheter in the area of the thrombus to achieve clot fragmentation and debulking. While the purpose of this technique is to remove a central obstruction and facilitate perfusion of downstream branches, thrombus fragmentation leads to distal embolization of small thrombi and may result in worsening pulmonary hypertension. ${ }^{46}$ Adjunctive therapy can also be performed with balloon catheter inflation to achieve fragmentation or aspiration through a large end-hole catheter.

There are numerous other devices for mechanical thrombectomy, though there are little data on the safety profile of these devices. ${ }^{18}$ The Aspirex is a rotational device that uses an Archimedean screw rotating within a catheter lumen to aspirate and remove thrombus. ${ }^{47}$ The Penumbra Indigo device is an aspiration device that applies continuous suction through an angled aspiration catheter of either 6 or $8 \mathrm{Fr}$ diameter. The device is delivered through a sheath in the PA; however, it does not use a guidewire which limits the operator's ability to direct the device into smaller PA branches. ${ }^{33}$ Additional devices, such as the AngioVac, FlowTriever, Argon Cleaner, and JETi Throm-
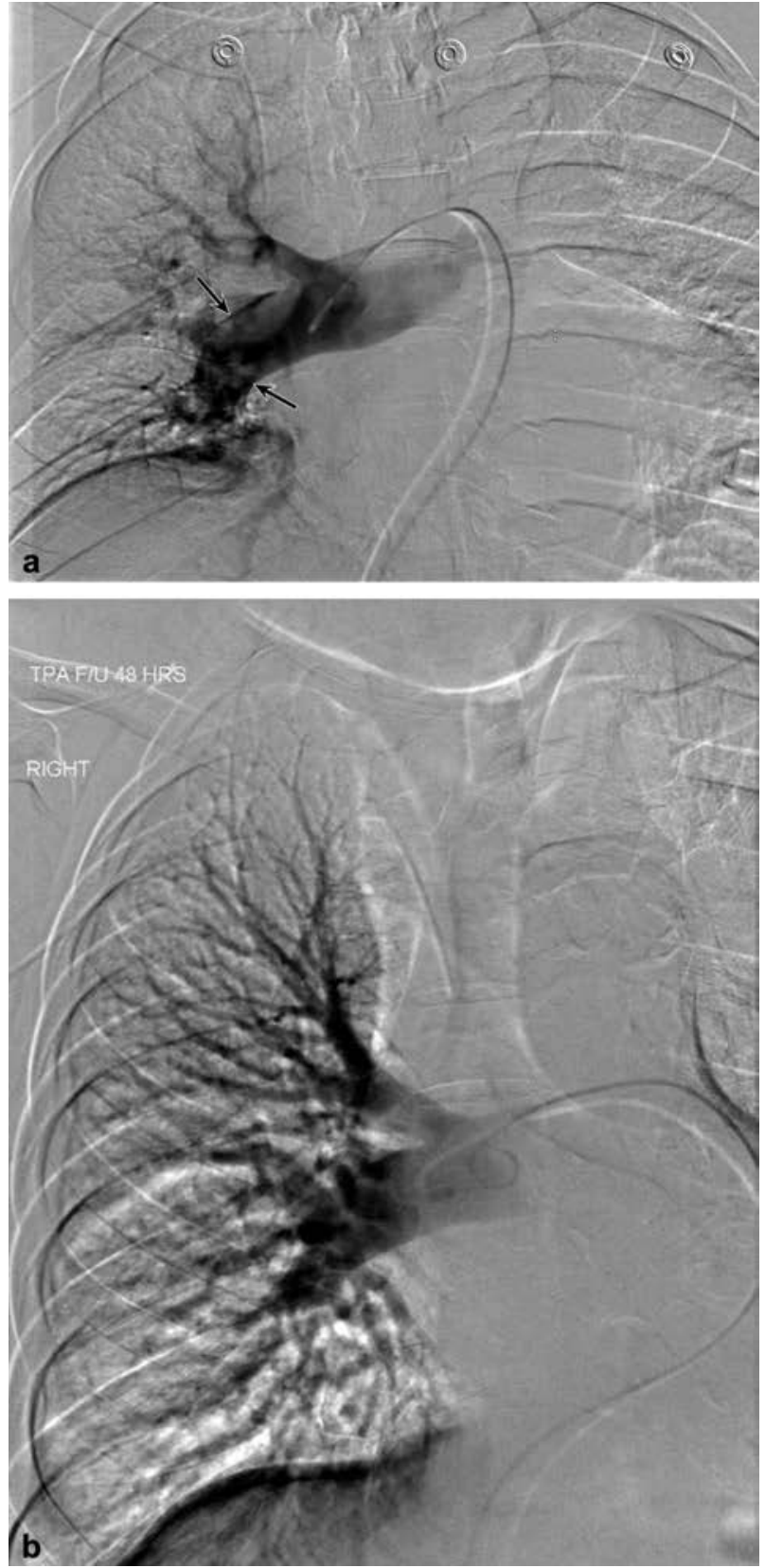

Fig. 4 Pulmonary angiography demonstrating findings before and after catheter-directed thrombolysis in a 68-year-old female patient who presented with submassive pulmonary embolism. (a) Multiple defects in the right pulmonary artery and its branches correspond with large clot burden (black arrows). (b) After 48 hours of catheterdirected thrombolysis, there is significant reduction of the pulmonary emboli, with no residual filling defects visualized. This corresponded with a reduction in the patient's measured pulmonary arterial pressures and clinical improvement.

bectomy System, have been used for CDT. With the exception of the FlowTriever device (FlowTriever received FDA clearance for the treatment of pulmonary embolism in May of 2018 based on results of the FLARE trial), none of these devices are currently FDA approved for use in the pulmonary circulation. ${ }^{33,37,46,48}$

The AngioJet (Boston Scientific, Marlborough, MA) is a rheolytic device that has become widespread in the treatment of deep venous thrombosis. Although initially promising for the treatment of acute PE, the AngioJet has been 
52 Sista AK. The OPTALYSE PE trial: another step toward understanding the truth about catheter-directed thrombolysis for submassive pulmonary embolism. JACC Cardiovasc Interv 2018;11(14): 1411-1413

53 Piazza G, Hohlfelder B, Jaff MR, et al; SEATTLE II Investigators. A prospective, single-arm, multicenter trial of ultrasound-facilitated, catheter-directed, low-dose fibrinolysis for acute massive and submassive pulmonary embolism: the SEATTLE II study. JACC Cardiovasc Interv 2015;8(10):1382-1392

54 Tapson VF, Sterling K, Jones N, et al. A randomized trial of the optimum duration of acoustic pulse thrombolysis procedure in acute intermediate-risk pulmonary embolism: the OPTALYSE PE trial. JACC Cardiovasc Interv 2018;11(14):1401-1410

55 Kuo WT, Banerjee A, Kim PS, et al. Pulmonary Embolism Response to Fragmentation, Embolectomy, and Catheter Thrombolysis
(PERFECT): initial results from a prospective multicenter registry. Chest 2015;148(03):667-673

56 Sista AK, Goldhaber SZ, Vedantham S, et al. Research priorities in submassive pulmonary embolism: proceedings from a multidisciplinary research consensus panel. J Vasc Interv Radiol 2016;27 (06):787-794

57 Mismetti P, Laporte S, Pellerin O, et al; PREPIC2 Study Group. Effect of a retrievable inferior vena cava filter plus anticoagulation vs anticoagulation alone on risk of recurrent pulmonary embolism: a randomized clinical trial. JAMA 2015;313(16):1627-1635

58 Dudzinski DM, Piazza G. Multidisciplinary pulmonary embolism response teams. Circulation 2016;133(01):98-103

59 Carroll BJ, Pemberton H, Bauer KA, et al. Initiation of a multidisciplinary, rapid response team to massive and submassive pulmonary embolism. Am J Cardiol 2017;120(08):1393-1398

This article has been corrected in accordance with the Erratum published on March 23, 2020. The Flow Triever device mentioned on page 68 is FDA approved. 